\title{
Écritures du monde, un centre de ressources culturelles et techniques pour les applications multi-écritures
}

\section{Vers une grammatologie universelle?}

\author{
Michel Bottin ${ }^{1}$ \\ Ministère de la culture et de la communication/DAG/DSI/MIVT \\ rue du Fort de Saint-Cyr, Montigny-le-Bretonneux \\ F-78182 ST-Quentin en Yvelines cedex \\ michel.bottin@culture.fr
}

Propos recueillis par Yvonne Sallé (ingénieur de recherche au CNRS)

RÉSUMÉ. Écritures du monde est un centre de ressources web créé par Monique Slodzian, François Stuck et Michel Bottin, consacré aux applications des écritures du monde. Il a pour ambition de donner accès à tout un contexte, tant technique que culturel, indispensable pour gérer des documents ou écrire dans des situations multi-écritures. Sachant qu'Unicode ne concerne que la codification universelle des caractères, il manque, en effet, à la majorité des utilisateurs tout un savoir culturel et technique qu'Écritures du monde leur offre. Ce site intègre donc des éléments de la généalogie et de l'histoire des écritures. Il décrit aussi le dispositif général d'Unicode et le contexte informatique élargi (moteur de recherche, polices, traitements de texte, tris alphabétiques et autres outils de gestion multilingues). Enfin, il décrit des savoirs précis : typographie traditionnelle ou informatique, translittération, calligraphie...

ABSTRACT. Écritures du monde (World Script) is a Web resource center created by Monique Slodzian, François Stuck and Michel Bottin, devoted to applications related to the writing systems of the world. Its ambition is to give access to the whole context, technical and cultural, necessary to manage documents or writing in multiscript environment. We know that Unicode is concerned by universal coding of all the existing characters but the greatest part of users do'nt have the cultural and technical knowledge offered by Écritures du monde. This web site is incluing elements of the genealogy and story of scripts. It describes also the general processing of Unicode and the broad data processing context (search engines, font, word processor, collating sequences and others tools). It describes also specific knowledges like traditional or computer typography, transliteration, calligraphy..

MOTS-CLÉS: système d'écriture, codage, caractère, glyphe, Unicode, police de caractère, TrueType, OpenType, chinois, arabe, vietnamien, latin, cyrillique, tamoul, inuktitut, graphème, grammatologie.

KEYWORDS: writing system, coding, caracter, glyph, Unicode, font, TrueType, OpenType, chinese, arabic, vietnamese, latin, cyrillic, tamil, inuktitut, graphem, grammatology.

1. Chargé de cours au CRIM/INALCO.

Document numérique. Volume $6-\mathrm{n}^{\circ} 3-4 / 2002$, pages 237 à 254 
YvonNe SALLÉ - Michel Bottin, vous avez réalisé un site internet au ministère de la Culture, intitulé Écritures $d u$ monde ${ }^{2}$. Pourriez-vous me préciser quel est le but de ce site sur les écritures du monde et comment l'idée vous en venue? Précédemment, vous aviez réalisé le site Écritures au Vietnam ${ }^{3}$. Écritures du monde s'inscrit-il dans la continuité du site Écritures au Vietnam ?

Michel BotTiN - Le site Écritures du monde est installé sur celui du ministère de la Culture, et hébergé par la Délégation générale à la langue française et aux langues de France (DGLFLF).

Il est développé en collaboration avec Monique Slodzian, responsable du Centre de recherche en ingénierie multilingue (CRIM), laboratoire de l'Institut national des langues et civilisations orientales (INALCO) de François Stuck et d'autres chercheurs du CRIM avec la participation d'enseignants de l'INALCO.

L'avènement des travaux de la norme ISO 10646 et d'Unicode permet enfin une représentation cohérente et uniforme des écritures du monde actuellement en usage ainsi que de quelques écritures anciennes. Chaque caractère de chaque écriture possède un code unique et distinct ce qui rend la codification des textes absolument non ambiguë donc leur circulation sûre et aisée. Cette situation tout à fait inédite dans l'histoire, mérite bien le qualificatif de révolution anthropologique si l'on en mesure les conséquences interculturelles et politiques.

Il est enfin possible d'envisager la diffusion de textes multilingues et multiécritures où toutes les écritures sont traitées sur un quasi pied d'égalité. Et cela est devenu réalité en ce qui concerne l'encodage donc le stockage; l'échange des fichiers multilingues au moyen de tous les protocoles est maintenant possible, du transfert de fichiers à la consultation de pages sur le web en passant par la messagerie électronique. Mais si cette égalité est pratiquement réalisée au niveau de la codification - et c'est le but que se sont fixés le standard Unicode et la norme ISO 10646 - beaucoup de travail reste encore à faire en ce qui concerne les différents visionneurs de textes (traitements de texte, navigateurs web, etc.), les polices de caractères, les méthodes de saisie.

L'ambition de ce site - loin d'être réalisée, il est vrai, pour l'instant - est de fournir un état de l'art, pratique et actualisé, de la représentation des textes dans les principales écritures du monde. Dans les faits, et c'est là toute la question, les problèmes qui se posent tant à des informaticiens qu'à des usagers professionnels de la communication ou à des utilisateurs au sens beaucoup plus large tels que les administrations, les milieux de l'enseignement ou des affaires, les spécialistes... sont complexes, appartiennent à des savoirs très divers allant de la linguistique à l'informatique la plus pointue en passant par la typographie... de telle sorte qu'il est pratiquement impossible à tout un chacun d'avoir une appréhension globale de ces savoirs divers.

2. http://www.culture.fr/edm/

3. http://www.culture.fr/evn/ 
Or, pour mettre en œuvre avec efficacité ces techniques à un niveau relativement global, par exemple, organiser un système d'information pour un centre d'affaires international ou un ministère des affaires étrangères, il faut disposer de ressources technoculturelles appartenant simultanément à tous ces savoirs. Ces savoirs sont culturels tels que les règles d'écriture des différentes langues écrites dans le monde mais ils comportent aussi des savoir-faire de type informatique.

YVONNE SALLÉ - Peut-on dire qu'Écritures au Vietnam a été une préfiguration du site Écritures du monde?

Michel BotTin - Écritures au Vietnam a été créé par Christiane Rageau et moi-même d'une manière pragmatique et sur une zone géographique très délimitée. Il a été présenté pour la première fois à l'occasion d'un sommet francophone qui se tenait à Hanoi en 1997. Son développement a servi quelque peu de modèle à ce qui est devenu, par la suite, le projet Écritures du monde. Mais Écritures au Vietnam est centré sur un territoire très particulier car particulièrement riche en écritures ce qui explique son intérêt au-delà de l'actualité du moment. En effet, le Vietnam a connu, au cours des siècles, un certain nombre de cultures d'écritures qui correspondent à des phases d'influences :

- d'abord l'écriture chinoise pour noter le chinois proprement dit ;

- puis une écriture en caractères chinois de la langue vietnamienne, le nôm, ce qui a conduit à l'invention de nouveaux caractères propres au Vietnam ;

- à partir du XVII ${ }^{\mathrm{e}}$ siècle, transcription du vietnamien en alphabet latin adapté grâce à l'ajout d'un certain nombre de signes diacritiques, d'accents et de marques afin de représenter les tonalités propres à cette langue ; ce travail a été réalisé par les missionnaires portugais à l'usage des étrangers afin de leur permettre de lire et d'écrire le vietnamien ;

- ce mouvement a été amplifié par la colonisation française et, à la fin de celle-ci, par les nationalistes qui y ont vu un mode de modernisation et de démocratisation de la culture ;

- parallèlement, le Vietnam comporte aussi de nombreuses minorités nationales qui avaient étés influencées culturellement par le monde indien et qui possèdent des écritures de type indien commes les écritures tai, cham, khmère. Il est à noter que d'autres minorités, dans le nord du Vietnam, utilisent aussi des caractères de type chinois pour noter leur propre langue.

Simultanément, l'écriture chinoise a continué d'exister en particulier à travers la communauté chinoise présente dans le pays, de même que l'écriture vietnamienne sous forme idéographique utilisée dans des catégories sociales très diverses. Ainsi, en 1930, on trouvait des tracts du parti communiste rédigés en écriture sinovietnamienne.

Du point de vue de l'informatique, la première phase de numérisation de la langue vietnamienne (avant Unicode) a été réalisée sous plusieurs standards 
concurrents élaborés par le Vietnam du nord, le Vietnam du sud et les différents groupes de la diaspora, soit près d'une vingtaine de «standards ».

C'est l'apport d'Unicode d'avoir unifié ces premiers standards. De plus, cas particulier intéressant, le Vietnam a rejoint le groupe de travail sur les caractères «idéographiques » chinois, tant et si bien que l'inventaire des caractères connu sous le sigle anglais CJK (pour Chinese, Japanese, Korean) s'appelle désormais CJKV !

Le site Écritures au Vietnam essaye de rendre compte de ce phénomène culturel ; il donne des documents photographiques et calligraphiques, des exemples de textes, de postures d'écriture, des formes particulières d'écritures (verticale, horizontale...). Au plan pratique, il indiquait comment accéder au site Unicode avec des renvois vers ce site, comment utiliser l'écriture vietnamienne; il donnait la classification des caractères latins et chinois avec un catalogue des clés de classification...

Ce site a donc été à l'origine de la conception du site Écritures du monde parce qu'il montrait que l'on pouvait résoudre les problèmes de ce type pour un lieu de confluences d'écritures.

YvonNe SALLÉ - Pouvons-nous revenir au site Écritures du monde pour voir comment il s'articule avec Unicode?

Michel BotTiN - Écritures du monde a l'ambition d'être un site encyclopédique de description de toutes les écritures du monde dans toutes leurs facettes d'usages, parce que l'on ne peut s'expliquer le fonctionnement réel de bien des langues que si l'on en connait les règles, l'histoire, la culture...

Il doit donc servir, en complément des tables techniques d'Unicode, à documenter culturellement et techniquement celui qui est confronté à une telle tâche. Ce site s'appuie donc directement sur Unicode.

Avec Unicode, on est arrivé à une codification universelle de toutes les écritures en usage dans le monde et même des écritures anciennes qui ne sont plus d'usage pratique ou en usage du tout. C'est une codification non ambiguë, c'est-à-dire que chaque caractère, dans chaque écriture, a un code distinct des autres caractères quelle que soit la langue : on n'a donc plus les problèmes que l'on avait auparavant lorsqu'on recevait un message que l'on savait, par ailleurs, être en chinois, en grec : on avait, certes, un message en code binaire mais son interprétation (sa visualisation) nécessitait, en plus, de savoir quel codage avait été utilisé, indication en général absente dans le message lui-même et souvent inconnue de l'auteur du document ! Et même si ces indications étaient connues, il fallait alors se mettre en quête de la police de caractères adéquate (c'est-à-dire utilisant le même codage que le message), l'installer sur le système d'exploitation si celui-ci pouvait effectivement la supporter. Cependant, toute cette normalisation de la codification des caractères, n'est en aucune manière suffisante pour répondre aux questions autres que strictement techniques et de plus limitées à la description des caractères des écritures du monde. La création du site Écritures du monde est donc indispensable. 
Supposons que quelqu'un, en France, ait besoin de lire des textes en arabe. Cette personne devra installer, sur son ordinateur, des programmes adaptés à l'écriture arabe; il y a plusieurs techniques pour cela et le site Écritures du monde devra répondre à ses interrogations.

Au-delà de cet indispensable savoir interculturel, ce webmaster devra, aussi, bien sûr, disposer de l'environnement technique et typographique propre à ces écritures qu'Écritures du monde lui indiquera aussi.

On peut imaginer, également, le cas d'une personne à qui il est commandé une nouvelle police de caractères. Quelle que soit sa compétence linguistique et technique, nul ne connaît toutes les écritures du monde. Or, c'est bien l'exhaustivité des règles de fonctionnement de toutes les écritures du monde qui sont requises pour pouvoir réaliser une police Unicode universelle ou des polices spécifiques à une ou plusieurs écritures.

YvonNe SAllé - Pouvez-vous préciser ce que vous entendez par toutes les écritures? Qu'en est-il des écritures rares ou anciennes?

Les tables de code ont été définies pour toutes les écritures des langues vivantes alphabétiques ou non; je citerai à titre d'exemple l'écriture latine, grecque, cyrillique, arménienne, géorgienne, hébraïque, arabe, les diverses écritures dérivées de la brahmi, chinoise, japonaise, coréenne sans parler d'écritures de moindre extension ni des écritures techniques comme les symboles mathématiques ou du langage de programmation (en particulier APL) ou des symboles graphiques divers. Les écritures anciennes qui ne sont plus utilisées sont entrées dans le même processus: la gothique et les anciennes écritures italiques (au premier chef l'étrusque) sont déjà codées ainsi que les signes musicaux byzantins. Les hiéroglyphes égyptiens ainsi que les écritures cunéiformes sont en route...

Bien entendu, cette codification universelle s'est réalisée d'une manière pragmatique : les experts se sont appuyés sur des codifications réalisées isolément pour diverses écritures dans les pays qui les utilisent ; on a donc repris des jeux complets de caractères codifiés et, en les déplaçant, on les a insérés dans Unicode

Les experts ont essayé de définir une base théorique en distinguant la notion de caractère de la notion de glyphe. Il existe une notion abstraite du caractère indépendante de sa réalisation graphique. Prenons, par exemple, la lettre «A». Le glyphe, c'est sa représentation graphique : on trouve, par exemple, le caractère " $\mathrm{A}$ » sous des formes différentes, en romain, en italique ou en gothique... il s'agit d'autant de glyphes; ou bien la forme différente d'une lettre selon la place qu'elle occupe dans le mot comme cela se produit en grec, en arabe... Il a donc plusieurs glyphes pour un même caractère.

Tout ceci a été défini, à l'origine, dans Unicode, toutefois on ne s'y est pas toujours tenu parce qu'on a repris des jeux de caractères existants dont l'analyse n'avait pas été aussi stricte : il en résulte un certain flou théorique mais qui, dans la pratique, n'empêche pas le système de fonctionner. 
YvONNE SALLÉ - Quel est l'intérêt de faire un tel travail de codification pour des écritures anciennes qui ne servent qu'à la recherche?

Michel BotTin - A partir des textes de Sumer, par exemple, les personnes qui lisent des textes en cunéiforme constituent des corpus de textes, développent des traitements d'analyse, élaborent des lexiques, des dictionnaires. Auparavant, il existait presque autant de systèmes de codification que de chercheurs qui les avaient conçus de sorte qu'il était très difficile de les échanger. Avec la recodification normalisée, chaque caractère s'est vu attribuer une valeur non ambiguë ce qui permet les échanges des textes entre chercheurs sans aucune difficulté. On comprend pourquoi la cohérence des corpus et la généralité des outils de traitement sont aussi étroitement liés à une codification universelle.

Pour illustrer ceci, je rappellerai que les Vietnamiens avaient réalisé une vingtaine de jeux de caractères codifiés, différents selon les groupes, selon les chapelles et cette cacophonie de codages bloquait les échanges. Au Cambodge, la situation était encore plus dramatique: en effet, chaque police de caractères correspondait à un codage particulier de sorte qu'ils en étaient arrivés à ne pas pouvoir échanger des fichiers d'un ministère à l'autre !

On voit donc très vite que ce problème de la codification des caractères, qu'on le considère au niveau d'un pays ou au niveau mondial, n'est pas seulement un problème technique, c'est aussi un problème politique. Cela explique pourquoi la mise au point d'une codification universelle a demandé autant de temps.

YvonNe SALlÉ - Puisqu'Unicode existe, comment voyez-vous la complémentarité entre le site d'Unicode et le site Écritures du monde?

Michel BotTin - Avec Unicode, actuellement, l'on dispose de près de 60000 caractères codifiés ; ils sont regroupés dans la norme ISO/CEI 10646/Unicode. Mais, outre le simple inventaire des caractères, beaucoup de détails sont encore nécessaires tels que :

- le nom normalisé du caractère (par exemple LATIN LETTER A UPPERCASE),

- sa directionnalité,

- les signes complémentaires qui peuvent l'accompagner,

- les propriétés du caractère dans ces différents systèmes,

- les clés de compréhension,

- la transformation des données à 32 bits d'Unicode en données à 16 bits et à 8 bits.

Les difficultés pour construire ce site sont énormes et de tous ordres. En premier lieu, il existe des types d'écritures très différents que l'on classe souvent en morphémographiques, syllabiques, néo-syllabiques, alphabétiques... sans pour autant être sûr de la pertinence ou des effets induits de cette classification en l'absence d'une véritable science de l'écriture ou grammatologie. 
En résumé, le site Écritures du monde sera un centre de ressources :

- donnant les éléments de description de l'écriture,

- donnant le minimum de ce qu'il faut savoir sur l'origine d'une écriture, soit un aperçu géographique et historique : origine culturelle et géographique, période de création...

- indiquant le fonctionnement : comment les signes se combinent entre eux pour que ça fonctionne, dans quel sens ils s'écrivent...

- indiquant quelles langues utilisent une même écriture, par exemple l'écriture arabe utilisée pour le persan, le turc ottoman, l'ourdou, le pashto, le kurde sorani...

- donnant un extrait de texte à titre d'exemple,

- indiquant quels sont les outils : traitements de texte, polices de caractères, navigateurs, outils informatiques pour saisir, afficher, faire circuler les textes concernant chaque écriture et comment diffuser sur le web des textes en toutes écritures et langues...

A propos de l'affichage, il est important de souligner les problèmes que soulève la directionnalité, problèmes qui doivent être résolus d'un strict point de vue informatique: citons l'écriture arabe qui s'écrit de droite à gauche mais dans laquelle les nombres s'écrivent de gauche à droite dans le cours du texte.

Ce travail présente peu de problèmes pour les écritures alphabétiques qui ont peu de caractères et peuvent être saisies directement au clavier. Un clavier est un dispositif d'une centaine de touches qui, même muni d'un système d'échappement comme l'usage de la touche «majuscule » (l'équivalent anglais «shift»= décalage, transposition indique mieux la nature de l'opération) ou de la touche « alt», permet la saisie de plus de deux ou trois centaines de caractères. Ceci peut donc convenir directement pour des écritures alphabétiques ou syllabiques. En revanche, pour les écritures chinoises qui comportent des milliers de caractères, il faudra la frappe de plusieurs touches du clavier pour saisir un seul caractère (parfois à l'aide même de dictionnaires) puisqu'il n'est pas question de réaliser des claviers comportant des milliers de touches. Ce procédé est connu sous le nom de méthode d'entrée (en anglais IME). Et des dizaines de telles méthodes ont été effectivement implémentées pour le chinois ou le japonais !

Par ailleurs, on constate que la linguistique ne nous a pas, jusqu'à présent, fourni de description formelle des systèmes d'écritures de même niveau que ce qui est donné dans les descriptions phonologiques ou syntagmatiques. Il est, d'ailleurs, intéressant de remarquer que la description d'une écriture est toujours difficile, que les linguistes interrogés aient ou non cette langue pour langue maternelle. En fait, ce travail d'analyse nécessite un niveau d'objectivation et d'abstraction toujours très difficile à accomplir.

Écritures du monde doit donc permettre de comprendre les principes de numérisation et de modélisation. Pour simplifier, on pourrait dire qu'il a deux aspects : 
- une facette informatique pour expliquer comment on utilise les caractères : leur saisie, leur stockage et leur transmission, les traitements d'ordonnancement (les tris), les divers procédés de rendu: affichage des caractères, direction de l'écriture (gauche à droite, droite à gauche, verticale, mixte...), les ligatures obligatoires ou simplement esthétiques, la segmentation éventuelle des mots ou des phrases en fin de ligne ;

- des aspects culturels précisant l'origine et le contexte d'emploi de tel ou tel aspect de l'écriture.

YVONNE SALLÉ — Qui sont les usagers d'un tel site?

Michel BotTin — La gamme des usagers peut être extrêmement large : on pense, bien sûr, en premier lieu, aux écoles de langues, aux centres culturels, aux grandes bibliothèques, aux linguistes, aux services des ministères des affaires étrangères, aux milieux d'affaires internationaux... Parmi d'autres catégories professionnelles, il y a les spécialistes de la localisation des logiciels (c'est-à-dire l'adaptation des logiciels créés dans un autre pays et dans une autre langue), les personnes qui dirigent des équipes ou des projets dans la cacophonie des langues et tous ceux qui sont confrontés à des problématiques d'informatisation dans un cadre multilingue plus ou moins « exotique ».

Yvonne SALlÉ - Pouvez-vous me dire comment vous planifiez cette généalogie des écritures? Avez-vous prévu des familles et pouvez-vous citer les principales familles que vous retiendrez ?

MiCHEL BOTTIN - A priori toutes les écritures! Mais leur regroupement pose des problèmes théoriques très vifs. Nous essayons donc d'avancer de la manière habituelle par les descriptions détaillées des écritures regroupées de manière plus ou moins traditionnelle. Ce n'est qu'ensuite que l'on pourra s'attaquer à la reclassification des écritures, c'est-à-dire à la critique de la classification actuelle.

D’une manière générale, nous avons décidé de nous consacrer, en priorité, aux écritures fortement liées à la notation d'éléments de langue (la nature de ce lien restant à articuler). C'est pour cela que nous avons repris la classification traditionnelle :

- d'écriture morphémographique, exemple l'écriture chinoise,

- d'écriture syllabique, exemple les kana japonais,

- d'écriture phonographique, exemples les alphabets latins, grecs, cyrilliques,

- les écritures néo-syllabiques, regroupements d'éléments phonétiquement motivés en agrégats graphiques de niveau syllabique, exemple, dans des univers scriptiques très différents, les écritures indiennes et coréenne.

Mais on est vite confronté à la quasi-inexistence de types purs : les systèmes d'écritures présentent toujours, à des degrés divers, un mélange de types dont seule l'histoire permet de rendre compte. De plus, l'élargissement aux écritures techniques, phonétique internationale, logico-mathématique, informatique, 
graphique, musicales s'impose si l'on veut donner toutes les dimensions du système scriptique.

Cependant la première version du site ne détaille qu'une seule écriture par type structurel :

- une écriture alphabétique-consonnantique : l'arabe,

- une écriture alphabétique : la cyrillique,

- une écriture morphémographique : la chinoise,

- une écriture néo-syllabique indienne : la tamoule,

- une écriture moins répandue, le Qaniujaaqpait, syllabaire inuktitut.

Les prochaines écritures en cours de description sont la latine, la grecque, l'hébraïque, la coréenne, la devanagari et la khmère.

YVONNE SALLÉ - Pouvez-vous, par quelques exemples, indiquer en quoi la généalogie et l'histoire des écritures peuvent éclairer les utilisateurs actuels ?

Michel BOTTIN — Nous venons de voir comment des systèmes d'écritures concrets sont des systèmes historiquement constitués. Mais, ce voile de l'histoire n'a pas seulement abouti à des dépôts internes au système d'écriture. Toute l'histoire « externe », culturelle, économique, politique est influencée, est remplie de légendes, de mythes ou d'anecdotes relatives à l'écrit. Et cela d'autant plus, si l'on considère l'écriture non plus comme glossographie stricte mais comme ensemble de signes non directement en correspondance avec des éléments de langue. L'aventure des écritures devient alors trace visible de la praxis humaine dont les témoignages archéologiques et ethnologiques s'étendent bien au-delà de ce que l'on veut bien considérer en Occident comme relevant de l'écriture.

Voici, par exemple, quelques histoires de divers ordres.

Pour le chinois, l'obligation de passer au pinceau a complètement changé l'écriture. A partir de ce moment, il existe un continuum entre la peinture et l'écriture : écrire c'est savoir dessiner, c'est pourquoi on peut inclure des textes dans les tableaux qui deviennent, dans certains cas, de véritables poèmes visuels dans lesquels dessin et sens des mots se complètent. En effet, en chinois, tout nom doit avoir un sens en rapport avec la chose nommée: le mot homme représente un homme d'une manière simplifiée, Chine signifie l'empire du milieu, Japon signifie l'empire du soleil levant... Vincennes est représenté par deux caractères wen et sen, qui signifient forêt des connaissances. Ce lien entre dessin et sens explique aussi pourquoi un petit Chinois, dès deux ans, est capable d'écrire le signe homme et quelques autres signes simples ; en sortant du lycée il en sait suffisamment pour lire un journal mais il devra toujours apprendre de nouveaux caractères surtout s'il veut étudier les textes anciens.

L'écriture chinoise viendrait, selon les légendes, soit des écailles de tortues que les devins faisaient brûler et qui prenaient alors différentes formes qu'ils ont reproduites, soit des traces laissées par un oiseau sur la neige. 
L'écriture japonaise est une écriture dérivée de la chinoise. Les Japonais ont emprunté les caractères du chinois vers le $\mathrm{VI}^{\mathrm{e}}$ siècle, avec une prononciation adaptée à leurs habitudes phonologiques de l'époque. Mais leur langue, contrairement au chinois, est une langue flexionnelle dont les morphèmes sont polysyllabiques. Le résultat en est que certains éléments de leur langue sont notés, soit selon le son, soit selon le sens auxquels ils ajoutent des flexions ce qui aboutit à un système très complexe. Au cours de l'histoire, on peut dire, en simplifiant, qu'ils sont arrivés à constituer deux syllabaires concurrents, l'un simplifié qui auraient été réalisé par des femmes, l'autre beaucoup plus complexe réalisé par des moines jaloux de l'initiative des femmes. Un de ces syllabaires est maintenant utilisé pour noter les morphèmes flexionnels, l'autre seulement pour la transcription des noms étrangers. Bel exemple d'écriture mixte !

L'activité de trace symbolique est présente, dès le magdalénien, en Europe et, dans les périodes équivalentes, en Afrique ou en Asie. A côté des représentations très réalistes d'animaux, on trouve quantité de signes gravés sur les parois mais aussi sur des os ou des cailloux comme au Mas d'Azil. Cette activité sémiologique est donc, bien entendu, antérieure à la naissance des systèmes d'écritures à Sumer ou en Égypte. Est-elle aussi ancienne que l'apparition du langage articulé ? Les deux sontils liés à l'apparition de l'Homo sapiens sapiens?

En tout cas, l'attention apportée par les archéologues aux signes non textuels montre que, déjà au Moyen-Orient ou dans les Balkans, routes de diffusion du Néolithique, il existe de nombreuses notations difficiles à interpréter mais semblant liées à des activités de comptage. Antériorité de l'écriture mathématique sur l'écriture glossographique ? Rôle du trait unaire et de ses répétitions comme matrice de la trace articulée?

YvonNe SALLÉ - Devant de telles complexités géographiques et historiques, ne prévoyez-vous pas d'insérer des cartes? On comprend bien que les atlas linguistiques existants ne suffisent pas pour rendre compte de cette histoire et de l'extension géographique de ces différentes écritures et de leur généricité.

Michel BotTiN - En effet, je prévois que des cartes préciseront l'apparition des quatre foyers d'écritures sumérien, égyptien, chinois et amérindien ainsi qu'une visualisation de leur diffusion jusqu'à l'époque moderne. Cette cartographie est très complexe en raison des recouvrements multiples sur un même territoire. Aujourd'hui, c'est un chantier ouvert.

YvonNe SALLÉ - Vous avez évidemment prévu de décrire langue par langue, ou famille de langues par famille de langues, ou encore en termes généraux pour l'ensemble des écritures, des sujets comme la saisie des caractères, l'affichage et la mise en page.

Michel BotTiN - Nous avons déjà évoqué que l'informatisation des écritures suppose de résoudre toute une série de problèmes : 
- les jeux de caractères : du Baudot, de l'ASCII et ses variantes nationales, des caractères 8 et 16 bits jusqu' à la grande unification de l'ISO 10646/Unicode,

- les problèmes d'affichage : type de polices, moteurs de rendu,

- les problèmes de ligatures et de directionnalité,

- les problèmes de segmentation et d'ordonnancement : délimitation et coupures de mots, lexies complexes, tri, etc.

Pour revenir aux problèmes de position et de direction, tous les textes ne commencent pas leur affichage par la position en haut et à gauche, la position du caractère suivant étant à la droite du caractère courant. Les écritures sémitiques sont généralement dirigées de droite à gauche en partant d'en haut à droite. Mais il en existe bien d'autres comme le chinois ou le japonais traditionnel, dirigés de haut en bas en partant d'en haut à droite, voire comme le tifinagh (berbère touareg) dirigé de bas en haut en partant d'en bas à droite. Il y a même des directions obliques ! Naturellement le mélange des écritures pose des problèmes tout à fait originaux comme la gestion du curseur indiquant la position courante sur l'écran.

La coexistence des directions vers la droite et vers la gauche commence à être correctement implémentée sur les systèmes d'exploitations récents (Linux, MacOS $\mathrm{X}$, Windows2000/XP). Il n'en est pas encore de même pour les directions verticales mais le travail de définition de leur gestion est très avancé dans le niveau 3 des spécifications de feuilles de style pour le web (CSS3) ce qui fait augurer de prochaines implémentations.

En ce qui concerne les problèmes de ligatures et d'agrégation de caractères, toutes les écritures n'utilisent pas des glyphes discrets. Certaines, comme l'arabe ou le devanagari, rattachent certains de leurs glyphes au précédent et/ou au suivant. Dans le cas du devanagari, comme dans celui des écritures dérivées comme elle de la brahmi, ce sont même de véritables agrégats de glyphes correspondant à peu près mais pas toujours exactement à une syllabe de la langue parlée. Si le traitement de ces caractéristiques est pris en compte au niveau du système d'exploitation, l'utilisateur a quelques chances de les voir devenir opérationnelles au sein de plusieurs applications (traitement de texte, navigateur web, indexeurs, etc.). C'est, par exemple, le support des polices OpenType développées par Adobe et Microsoft qui offre ces possibilités grâce à l'existence des tables de substitution de glyphes GSUB qui permettent d'automatiser le choix et la position d'un glyphe en fonction du contexte.

YvonNe SAllé - Pouvez-vous, par quelques exemples, préciser des notions telles que calligraphie, rendu d'écriture, police de caractères... ?

Michel BotTin - On a bien un codage de toutes les écritures mais nous ne disposons pas vraiment de plate-forme informatique où toutes les écritures sont traitées de manière équivalente. C'est à ce propos que je parle de la nécessité de résoudre les problèmes de saisie, d'affichage ou de moteurs de rendu, notions en 
général peu compréhensibles en dehors du monde des informaticiens. C'est cependant capital pour la pratique.

De plus, ce discours est obscurci par les pratiques préexistantes à l'Unicode. On constate que des spécialistes de diverses langues ayant souvent fabriqué leurs propres systèmes sont, de ce fait, amenés à penser qu'Unicode ne peut pas fonctionner. Pour l'écriture khmère, ils en avaient convaincu le gouvernement cambodgien qui s'était rallié à cette opinion par orgueil national. Unicode par sa mise en cause de travaux antérieurs a suscité l'apparition de résistances soit nationales, soit de la part des spécialistes. L'enjeu si l'on veut chercher à résoudre le cas général plutôt que des cascades de cas particuliers est de dégager ce qu'il y a de formellement commun entre certains systèmes d'écritures indépendamment de toutes considération idéologique ou partisane. Par exemple si l'on arrive à trouver un algorithme commun (avec éventuellement quelques variantes locales) pour toutes les écritures de type indien y compris la birmane, la thai, la khmère... ce serait un grand progrès. Mais pour cela il faut rentrer dans la cuisine du fonctionnement de l'écriture et c'est ce que souhaite décrire le site Écritures du monde: montrer comment ça marche, comment ça se décompose, comment on peut avoir une description formelle afin d'élaborer des algorithmes formels et qui pourrait permettre aux gens de comprendre pourquoi ça marche ou pas avec telle police de caractères et/ou telle système d'exploitation.

La codification est nécessaire mais n'est pas suffisante : en dehors du codage pas de salut ! Mais il faut, en outre, traiter la direction, les ligatures... pour que les textes soit tout simplement lisibles. Si l'on veut faire de la typographie soignée, voire de la calligraphie, c'est beaucoup plus difficile; cela dépasse, certes, l'objectif que s'étaient fixé les experts d'Unicode mais ce serait indispensable dans Écritures $d u$ monde. Or, les calligraphies de différentes cultures d'écritures obéissent à des économies très différentes. Que l'on songe simplement à la calligraphie arabe par opposition à la calligraphie chinoise !

Faire une police de caractères nécessite un travail long et minutieux : pour une police latine de qualité, on peut considérer qu'il faut environ six mois de travail pour un développeur mais plus encore pour une police tibétaine un tant soit peu complète. Il faut non seulement représenter les caractères, mais il faut encore régler les espaces entre les signes, les positionner au-dessous, au-dessus, devant...

Pour l'écriture khmère, pour faire le rendu de l'écriture qui est très compliquée, il faut 4 ou 5 étapes : on prend le caractère, on en cherche la forme quand il est lié puis il faut un programme pour faire le placement au bon endroit, dessus, dessous... C'est un savoir figé nécessitant un algorithme pour la police de caractères afin de fixer toutes ces règles. Ensuite, quand on charge une police de caractères, toutes ces règles d'écriture doivent y être incluses implicitement. Mais pour créer ces automates, il faut quelqu'un qui sache parfaitement comment fonctionne l'écriture et sache le décrire et le programmer à la main: ainsi, quand, au clavier on va taper successivement les lettres du khmère, on aura le dessin empilé de chaque caractère 
comme on le fait traditionnellement à la main. Bien entendu, il n'est pas question de réformer les écritures même si l'on estime qu'elles sont difficilement adaptable à l'informatique.

Ainsi au Cambodge, il n'y a pas d'uniformité du tout. Plusieurs systèmes ont été créés mais aucun n'a la même méthode. Il est donc très mauvais de tenter de s'inspirer de ce qui se faisait sur place parce qu'ils essayaient de le faire dans l'ordre visuel. Or, quand on a un texte en mémoire et que l'on veut créer l'index correspondant, par exemple, on ne peut pas utiliser l'ordre visuel. Un dictionnaire ne se classe pas dans l'ordre visuel mais dans l'ordre phonique. Prenons un exemple : dans la langue khmère, la syllabe kri s'écrit dans l'ordre rki. Quand on va la classer, il faut le faire à « $\mathrm{k}$ » et non à « $\mathrm{r} »$. On voit bien que pour faire des dictionnaires, il faut les coder dans l'ordre de la prononciation mais au moment où l'on va afficher le texte, il faut que les caractères apparaissent dans l'ordre habituel visuel de lecture ; il y a donc un petit moteur informatique qui va faire ce travail de représentation ce qui explique que les polices de caractères soient si compliquées.

En arabe, même si l'on note les voyelles, on n'en tient pas compte pour faire un tri alphabétique si ce n'est à un niveau secondaire. En plus, en ce qui concerne les polices, la difficulté vient de ce que la forme d'une lettre varie selon son environnement : donc la police doit pouvoir faire correspondre à un code donné, tel ou tel glyphe (forme graphique d'un caractère) suivant le contexte. Il s'ajoute à cela que le caractère suivant doit en principe être affiché à la gauche du précédent ; mais si la phrase comporte un nombre, par exemple 124, le 2 doit être affiché à droite du 1 et le 4 à la droite du 2 alors qu'en mémoire ils sont dans l'ordre 1,2,3. Cela suppose un traitement informatique complexe. Ce que le jargon d'Unicode décrit sous le nom de bidi pour bidirectionnel.

Pour le chinois, le problème est différent et beaucoup plus facile : il y a beaucoup de caractères mais il est simple d'afficher les traits dans le carré idéal de chacun d'eux. En outre, le caractère suivant s'affichera à la suite à droite ou en dessous. Il n'a pas de véritable complexité de ce côté-là.

YVONNE SALLÉ — Dans le site Écritures au Vietnam, il était fait une large part à l'iconographie. En sera-t-il de même dans Écritures du monde?

MiCHEL BotTiN - Concernant toutes les pratiques d'écritures, il existe une iconographie extrêmement abondante qu'il serait intéressant d'insérer dans le site. La culture typographique, par exemple, dont on trouve des illustrations anciennes dans les vingt-cinq planches de l'Encyclopédie de Diderot présentant les différents alphabets, syllabaires ainsi que les clefs chinoises sont un bon exemple de ce qu'il est prévu de mettre en ligne. Les difficultés concrètes proviennent seulement des moyens nécessaires pour effectuer une sélection de l'iconographie et la gestion des droits afférents aux images. Mais l'orientation générale restera plutôt du côté technique déjà très difficile à couvrir en lui-même. 
Yvonne Sallé - Dans le cadre d'Écritures du monde, quelles sont les difficultés que présente la description d'une écriture ?

Pour chaque écriture, il est prévu de donner un certain nombre d'éléments dont nous avons déjà parlé :

- un aperçu historique et géographique (genèse et extension),

- une description structurelle du fonctionnement de l'écriture,

- la liste des langues qui l'utilisent comme notation,

- un texte avec traduction dans une langue utilisant l'écriture donnée,

- les polices de caractères qui supportent cette écriture,

- les outils de saisie et d'affichage, tout particulièrement sur le web.

Toutefois le travail technique et informatique est considérable car, par exemple, les deux derniers éléments nécessitent une veille technologique très difficile à assumer.

Sur un autre plan, les premières descriptions des écritures - même sommaires ont fait apparaître l'absence d'un cadre général de description des systèmes d'écritures un tant soit peu formalisé. La notion d'élément de code d'une écriture ou caractère du standard Unicode, si elle va bien dans le bon sens, ne se révèle pas suffisante pour lui donner un statut théorique vraiment universel. D'autant plus que des considérations pragmatiques, au demeurant tout à fait louables, comme la compatibilité avec des encodages préexistants, ont fait entériner d'anciennes descriptions dont la cohérence formelle n'était pas toujours la caractéristique dominante.

De plus, nous nous sommes aperçus de la difficulté qu'avaient les différents collaborateurs du site à dégager le fait scriptural tant des faits linguistiques correspondants que de l'approche purement technicienne de la typographie ou de l'informatique. Comme si ce domaine ne possédait pas des caractéristiques propres ayant une autonomie véritable par rapport à des sciences et techniques connexes.

La description d'une écriture se révèle très vite être une source de difficultés ; en effet, on n'a pas de vocabulaire ou même de concepts universels pour décrire les éléments des écritures. Au mieux, on dispose de concepts locaux sur chaque écriture. Par exemple, que veut dire l'expression écriture de type alphabétique? Qu'est-ce qu'un alphabet consonnantique? Est-ce un syllabaire dont on ne note pas les voyelles ou s'agit-il d'un alphabet ? Ou n'est-ce qu'un nouvel avatar de la question du verre à moitié plein et du verre à moitié vide ?

On peut considérer que, dans un mode de pensée syllabique, comme au MoyenOrient, c'est le fait que les voyelles n'étaient pas essentielles pour la notation de la langue qui a permis aux Sémites, d'une certaine manière, d'inventer l'alphabet sans peut-être s'en rendre compte ; ce serait alors les Grecs qui auraient consciemment développé l'alphabet. Nous appelons maintenant, les alphabets sémitiques alphabets consonnantiques. 
De même, il existe des néo-syllabaires qui sont des systèmes analytiques qui fonctionnent structurellement comme des alphabets même si leur représentation de surface les fait ressembler à des syllabaires. Leurs usagers fonctionnent dans cet univers syllabique et admettent non sans résistance l'interprétation analytique avancée par l'Unicode pour le codage de leurs écritures.

On voit donc qu'il n'existe pas de description universelle.

J'ai donc recherché du côté des graphèmes. Le dictionnaire Larousse définit ce terme: "Unité graphique minimale entrant dans la composition d'un système d'écriture » mais on ne dit pas comment se définit une « unité graphique minimale ». On dispose des règles de connotation pour la description des phonèmes : si, en changeant de son on change le sens de la phrase ou du mot, il y a un phonème. Par exemple, en allemand, un phonème peut avoir deux réalisations quand le $c h$ se prononce dur ou doux selon le contexte ou la voyelle qui précède comme dans Buch et Chemie.

De fait, la définition du graphème est si générale qu'elle ne peut pas être fausse, mais pour une science elle est trop imprécise pour être opérationnelle.

Dans Pour une théorie de l'écriture de I. J. Gelb (Gelb, 1973) on s'attendrait à trouver des définitions mais il s'agit plutôt d'une analyse historique et diachronique depuis Sumer et d'une tentative pour remplacer les écritures idéographiques par des logogrammes. Or, le caractère chinois est plutôt un morphème.

O. Ducrot et T. Todorov dans leur Dictionnaire encyclopédique des sciences du langage (Ducrot et Todorov, 1972) donnent des définitions de base et parlent d'écritures morphémographiques, mais personne n'a vraiment travaillé sur le sujet même et ce malgré le développement d'Unicode. Il en résulte donc que nous n'avons toujours pas vraiment de terminologie ni de concepts assurés.

En interrogeant le terme graphème, sur internet, on trouve toutes les définitions possibles et imaginables mais aucune n'est satisfaisante: elles sont vagues ou inopérantes, ou bien s'appliquent aux alphabets latins, mais ne conviennent pas ou négligent le chinois...

Revenant au graphème, si l'on dit que les éléments de l'écriture sont des graphèmes, ceux-ci doivent-ils se définir par rapport au phonème ou y a-t-il des éléments de l'écriture qui permettent de définir le graphème sans référence à la langue parlée ? A l'examen, on constate qu'il est évident que le graphème est également lié à la langue et non seulement à l'écriture. De ce fait, il perd en universalité. Pour les alphabets, il est sûr que c'est lié à la phonologie car les écritures alphabétique sont, en quelque sorte, de niveau phonologique. C'est différent en chinois où le caractère correspond bien à un graphème. En revanche pour les écritures indiennes, on peut se poser la question : la syllabe graphique estelle un graphème ou bien faut-il la décomposer? Les contre-exemples étant possibles, faut-il revenir aux langues parlées pour définir le graphème ? Cela va dépendre des langues. 
Si l'on examine les règles de coupures de mots en fin de ligne ou les règles initiales de classement alphabétique (par exemple, dans les dictionnaires espagnols les mots commençant par $c h$ ou $l l$ ne sont pas classés aux lettres $c$ ou $l$ mais font l'objet d'un classement spécifique) on voit donc que, même pour les langues d'écriture latine, un graphème ne se confond pas complètement avec un caractère puisqu'il existe des graphèmes composés de deux ou plusieurs caractères. En français, la lettre $e$, lorsqu'elle est accentuée donne lieu, dans les lexiques à des tris secondaires, les mots commençant pas be étant placés avant ceux commençant par bé puis $b \hat{e} . .$. Ces règles sont indiquées avec beaucoup de précision dans les codes typographiques. Dans les autres langues basées sur l'alphabet latin et comportant des signes diacritiques, les exemples se multiplient. On n'a pas la réponse, mais les règles de coupure de mot et de tri d'ordonnancement suffisent-elles à définir les graphèmes ?

Dans les langues arabes, la notion de graphème est encore plus complexe à définir en raison des ligatures entre les lettres à l'intérieur des mots.

Tous ces exemples soulignent, à l'évidence, la nécessité d'une grammatologie universelle.

D'où la question récurrente : une science de l'écriture ou grammatologie est-elle possible ? Même si l'on se focalise sur l'écriture comme système sémiologique destiné à représenter des faits de langue, beaucoup de questions tout à fait fondamentales ne semblent pas abordées. Par exemple, les systèmes d'écritures sontils des systèmes formels dont on pourrait rendre compte sans avoir à recourir à la linguistique des langues parlées correspondantes ou doit-on recourir nécessairement aux éléments linguistiques qu'ils représentent? Peut-on donner une définition universelle du concept de graphème par des procédés de commutation d'éléments purement graphiques ou doit-on systématiquement passer par les référents de la langue parlée ? Mais en même temps, un système formel est lui-même un système d'écriture. Comment alors rendre compte par l'écriture d'un système d'écriture ? $\mathrm{Ne}$ sommes-nous pas dans l'aporie déjà abordée par Gödel à propos des limites des systèmes formels ?

En d'autres termes, la grammatologie est-elle susceptible d'une description indépendante du reste de la linguistique - quitte à décrire séparément l'articulation entre les deux plans - ou est-elle subordonnée à la phonologie et/ou à la morphologie ? Et l'on comprendra l'importance de cette question puisque la nature des réponses que l'on peut y apporter conditionne la formalisation.

A titre d'illustration et de réflexion sur le sujet, je livrerai ici quelques définitions des termes graphème, lettre et caractère. On y verra bien souvent le poids encore trop présent de l'européocentrisme alphabétique. 


\section{Graphème}

- Larousse :

Unité graphique minimale entrant dans la composition d'un système d'écriture.

- Le Robert :

La plus petite unité distinctive et significative de l'écriture, lettre ou groupe de lettres correspondant à un phonème ou à un repère morphologique, étymologique (exemple $c h$ en français).

-Gleason:

Un système d'écriture est constitué par un ensemble de graphèmes auquel s'ajoutent certains traits caractéristiques de leur emploi. Chaque graphème peut avoir un ou plusieurs allographes. Les graphèmes et les allographes ont, dans le système d'écriture, une situation comparable à celle des phonèmes et des allophones dans le système phonologique.

\section{$[\ldots]$}

Chaque graphème représente une certaine portion de la structure de la langue parlée associée ou sous-jacente. Cette portion constitue la référence du graphème. Puisque l'expression de la langue parlée consiste en deux systèmes structurels fondamentaux, il y a deux grands types de graphèmes du point de vue de leurs références. Le type de graphème le plus familier est celui qui a une référence phonologique. Un second type de graphème a une référence morphologique. Les graphèmes à référence morphologique sont généralement appelés idéogrammes.

- Lafont :

Graphe [trace modélisée] constituant une unité dans un système stabilisé.

\section{Lettre}

- Robert :

Signe graphique qui, employé seul ou combiné avec d'autres, représente, dans la langue écrite (écriture alphabétique, syllabique), un phonème ou un groupe de phonèmes.

\section{Caractère}

- Le Robert :

Signe gravé ou écrit, élément d'une écriture.

Ces quelques définitions, glanées ça et là, se caractérisent soit par une trop grande généralité qui leur ôte tout caractère opératoire soit par une trop grande restriction, implicite ou explicite, à l'univers alphabétique occidental. 


\section{Conclusion}

Nous allons continuer le développement de ce site destiné à recueillir les éléments descriptifs et explicatifs. Mais toutes les personnes intéressées à fournir la description d'une écriture donnée seront naturellement les bienvenues. La ligne graphique sera complètement renouvelée et beaucoup de pages statiques seront remplacées par des éléments issus dynamiquement du système SDX (système documentaire en XML) de manière a assurer une plus grande souplesse de présentation et une plus grande évolutivité des données.

Le travail théorique esquissé ci-dessus devrait être abordé au cours de l'année scolaire 2002-2003 au CRIM. Les travaux correspondants seront publiés sur le site web. De plus, un forum devrait être ouvert permettant de débattre de l'ensemble de ces questions entre toutes les personnes intéressées.

\section{Bibliographie, sites web}

Coulmas, Florian, 1989, The writing systems of the world, Oxford, Blackwell.

Ducrot, Oswald, Todorov, Tzvetan, Dictionnaire encyclopédique des sciences du langage, Paris, Seuil.

Février, James G., 1959, Histoire de l'écriture, Paris, Payot.

Gelb, I. J., 1973, Pour une théorie de l'écriture, Paris, Flammarion cop. (Idées et recherches) Trad. de : A study of writing, Chicago, Ill, Univ. Of Chicago, 1952.

Gleason, H.-A., 1963, Introduction à la linguistique, trad. de Françoise Dubois-Charlier, Paris, Larousse, (Sciences humaines et sociales).

Haas, William, Writing: the basic option, http://lings.ln.man.ac.uk/More/Haas/Art\%20of\%20Literacy/writin 1.htm

Haas, W., éd. 1976, Writing without Letters, Manchester University Press.

Jensen, Hans, 1969,Die Schrift in Vergangenheit und Gegenwart, G. P. Putnam's Sons.

Lafont, Robert (sous la dir. de)1984, Anthropologie de l'écriture, Robert Lafont, Paris, Centre Georges Pompidou, Centre de création industrielle (Alors).

Lloyd, M. A. Inventory of writing systems http://gurpsnet. sjgames. com/Archive/AdsDisSkills/Languages

Lunde, Ken, 1999, CJKV Information processing, Beijing, Cambridge, Köln, Paris, O’Reilly.

Rageau, Christiane, Bottin, Michel. Écritures au Vietnam, http://www.culture.fr/evn/

Sampson, Geoffrey, 1985, Writing systems: a linguistic introduction,Stanford, CA, Stanford Press.

Slodzian, Monique, Stuck, François, Bottin, Michel, Ecritures du monde, http://www. culture. fr/edm/ 